\title{
Method for Reduction the Errors of Likelihood in the Authentication by the Iris
}

\author{
Dmitrii V. Orel \\ Department of Organization and \\ Technology of Information Protection \\ North-Caucasus Federal University \\ Stavropol, Russia \\ kde.def@gmail.com
}

\author{
Aleksandr P. Zhuk \\ Department of Organization and \\ Technology of Information Protection \\ North-Caucasus Federal University \\ Stavropol, Russia \\ alekszhuk@mail.ru \\ Anna G. Vanina \\ Department of Organization and \\ Technology of Information Protection \\ North-Caucasus Federal University \\ Stavropol, Russia \\ hnykina_anna@mail.ru
}

\author{
Tatyana V. Minkina \\ Department of Organization and \\ Technology of Information Protection \\ North-Caucasus Federal University \\ Stavropol, Russia \\ n.min@mail.ru
}

\begin{abstract}
The article describes the influence of lighting on the accuracy of users authentication in access control systems on the iris. The low contrast of the iris image is the reason that increases the number of errors because of different lighting at the stages of registration and authentication of the user. Depending on the wavelength of light in which the iris is registered, various details appear on it, and their severity depends on the type of eye. Most light eyes give the clearest picture in visible light. On the contrary, the structures of dark eyes clearly appear in the infrared range. It is recommended to use an iris biometric authentication system with the functionality of visible and infrared scanning ranges. Then the system should evaluate the quality of the images obtained in the visible and infrared scanning ranges and select the best quality. This lead to the addition of a new step in the recognition algorithm, which increases the running time of the algorithm as a whole. To compensate the time required for that, it is recommended to use an optimal set of modules of residual classes system, which will improve the performance and technical characteristics of digital filters.
\end{abstract}

Keywords-iris, biometrics, control system, authentication, filtering, False Rejection Rate, visible lightning, infrared lightning, residual classes system.

\section{INTRODUCTION}

To date, the use of password authentication does not provide the appropriate level of security. To improve identity authentication systems the biometric characteristics are used, which ensure the protection level not lower than the usual systems with a password. The advantage of biometric authentication systems is a condition for the applicability of which does not allow to use a password to third parties. To minimize the risk of unauthorized use of a password to third persons are widely used methods of biometric authentication. One of the promising methods for biometric authentication is a method based on the authentication of an iris.

According to the analytical study of the company «Tractica», recent technological advances have opened a new field of application of biometric authentication by iris in consumer applications. According to analysts of the global market for biometric authentication systems for iris to grow to $\$ 4.1$ billion in 2025 [1].

According to the forecast of the company «Tractica», client authentication using biometric systems, including the iris become the main option of the technology in mobile devices, automatic teller machines (ATMs), etc.

On average, the market Biometric technology will grow by $22.9 \%$ every year. During the ten-year period the total turnover of the market will be about 69.8 billions of dollars.

Thus, the iris authentication is increasingly being used in everyday life. This means that it is necessary to improve the method and implementing it in the iris authentication algorithm to improve its performance and reduce the likelihood of errors.

The aim is to develop recommendations to improve the performance and reduce the likelihood of errors for authentication of an iris.

\section{METHOD DEVELOPMENT}

An important factor for building the authentication algorithm of the iris is the knowledge of the structure of the eye. The human eye is a spherical body is almost entirely covered with sclera - solid opaque shell. General structure of the human eye structure is shown in Fig. 1.

Transparent the anterior wall is called the cornea. Then there is the iris, which is a thin vascular plate and acts as a diaphragm that restricts the flow of light entering into the eye. The outer contour is the border of the iris to the sclera and is called limb. The inner boundary of the iris pupil is represented, which narrows in strong light, and expands with gentle.

The remaining elements of the eye disposed in its depth will not be considered in this article is not used as in the authentication process of the iris [2]. 


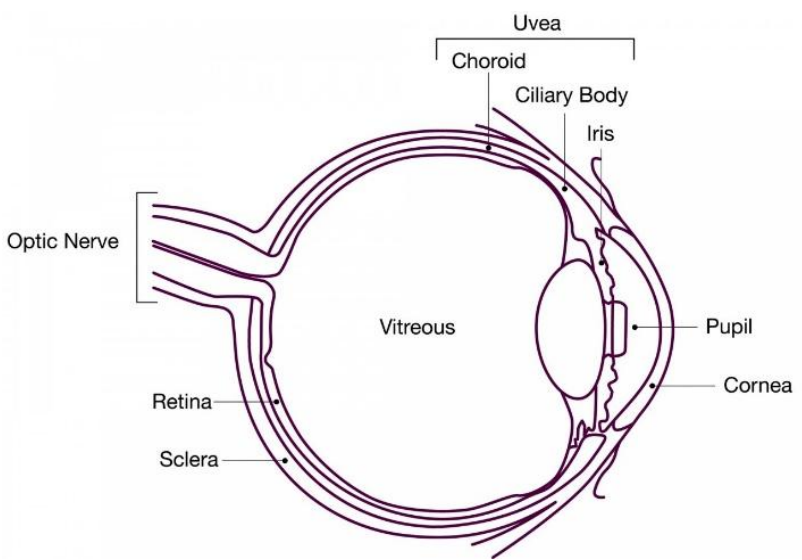

Fig. 1. The horizontal section of the right eye

Figure iris driven radially arranged filaments (trabeculae) intertwined with each other connective beams extending in different directions, and is unique to each person. Figure iris most people low contrast (range brightness image of the iris of points is much smaller than the brightness range of the image the eye, including the pupil dark and bright sclera). In addition, some types of iris have a fine texture (they do not have large bright or dark elements). This puts high demands on the image recording system. Depending on the wavelength of the light, which is registered iris, therein are shown various parts, and their expression depends on the type eye. For example, most light eyes gives the most clear picture in visible light. In the transition to infrared this figure gradually blurred and almost disappears at wavelengths of 900nm large. In contrast, dark eye structures are practically invisible in visible light, but clearly seen in the infrared. Therefore, the question as to which wavelength is optimal, still remains not decided [3].

When developing an authentication system for iris must carefully approach the issue of light reception area. Given the need to choose the right lighting, consider scenarios change in image quality in the bright and low light conditions.

\section{A. Scenario 1}

Consider importance saturation capacitance and dark noise in the photography under bright lighting by $(1-4)$ :

$$
\begin{aligned}
& \mathrm{SNR}=\frac{\text { Signal }}{\text { Noise }}=\frac{\text { number of electrons }}{\text { darknoise }+\sqrt{\text { number of electrons }}}= \\
& =\frac{n_{e^{-}}}{D N+\sqrt{n_{e^{-}}}}, \\
& \mathrm{SNR}=\frac{n_{e^{-}}}{D N+\sqrt{n_{e^{-}}}}=\frac{30000}{5+\sqrt{30000}} \approx 168.3 \\
& \mathrm{SNR}=\frac{n_{e^{-}}}{D N+\sqrt{n_{e^{-}}}}=\frac{30000}{10+\sqrt{30000}} \approx 163.8 \\
& \mathrm{SNR}=\frac{n_{e^{-}}}{D N+\sqrt{n_{e^{-}}}}=\frac{15000}{10+\sqrt{15000}} \approx 113.2
\end{aligned}
$$

where $n_{e^{-}}$- number of electrons; DN - dark noise.

It is evident from the research that, in the shooting a bright dark noise plays a secondary role. Even if double dark noise as in formula (3), the ratio "S / N" drops by only 3\%. However, if the number of stored electrons will be half (e.g., due to a lower saturation capacitance), the ratio "S / N" to decrease by $31 \%$.

\section{B. Scenario 2}

Consider importance dark noise in shooting under low illumination by the formulas $(5-6)$ :

$$
\begin{aligned}
& \mathrm{SNR}=\frac{n_{e^{-}}}{D N+\sqrt{n_{e^{-}}}}=\frac{50}{10+\sqrt{50}} \approx 4.1 \\
& \mathrm{SNR}=\frac{n_{e^{-}}}{D N+\sqrt{n_{e^{-}}}}=\frac{50}{10+\sqrt{50}} \approx 2.9
\end{aligned}
$$

It is evident from the research that if shooting in extremely low light conditions, the dark noise of great importance. For example, by doubling the dark noise in the formula (6) the ratio " $\mathrm{S} / \mathrm{N}$ " is reduced by approximately $30 \%$. When this saturation capacity in low light plays no role because the number of generated electrons is much less than the saturation point.

According to various research it can be concluded that in bright light important high saturation pixel capacitance, while the level of dark noise plays a minor role, and thus, an image registration is enough to use radiation in the visible range of the spectrum. In low light conditions, the saturation capacity is unimportant, whereas the level of dark noise is becoming increasingly important, therefore, to obtain a high quality image it is necessary to use infrared radiation.

To compare images obtained in two ranges - the visible and infrared, base used iris biometric data [4]. For images taken in the visible light spectrum, as shown in Fig. 2, the error of the first kind False Rejection Rate (FRR) was $77.1 \%$, against $14.4 \%$ - in the infrared (IR) range, as shown in Fig. 3. With that said substantial decline in quality in the visible spectrum recognition light for the entire system.

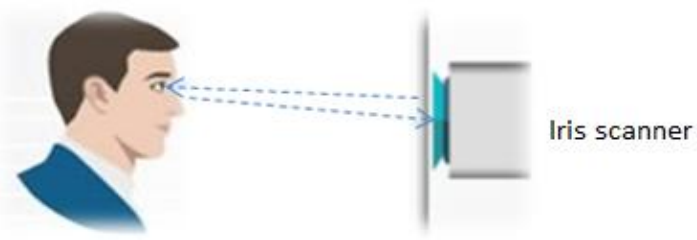

Fig. 2. Vector eye image registration laser emission range of the visible spectrum 


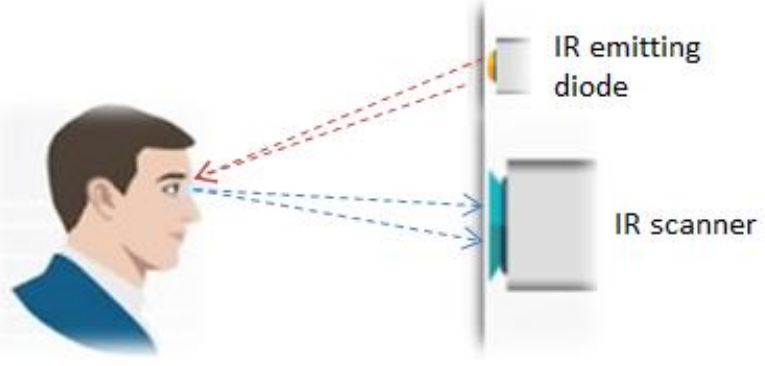

Fig. 3. Vector eye image registration IR laser light range of the spectrum

Thus, for the scanning radiation pattern iris is used most often close to the infrared range [5]. It is, first of all, allows the scanner to work even in the dark, and secondly, reads the picture is much more accurate than the radiation of the visible spectrum of light.

It should not be missed that in addition to lighting, you need to take into account the colour of the eyes of the person passing authentication. For example, the texture of the iris in people with dark eyes is poorly distinguishable in the visible range at registration, and vice versa, the texture of the iris in people with light eyes will be poorly distinguishable in the infrared range [6].

\section{RESULTS}

To capture the necessary elements of an iris in an image recognition system must have the possibility for at least 70 permitting pixels within a radius of the iris. To date, under normal conditions for the radius of the iris envelope characteristic resolution pixels 80-130. Monochrome CCD (charge-coupled device) camera $(480 \times 640)$ are used mainly as required for imaging illumination in the near infrared in the band of 700-900 nm, which unobtrusively for humans [7]. On some platforms, imaging deployed narrow-angle camera for coarse localization of the eyes in faces, to control the optics is also used narrow-angle camera pan and tilt, which receives the eye image with a higher resolution.

Focusing of the image is performed in real time (faster than the frame rate video) [8] by measuring the spectral power in the middle and upper frequency bands of twodimensional Fourier spectrum of each image frame and maximize this quantity, or by moving the active lens, or by sonic treatment to a subject for to appropriately adjust their range. Execution speed assessment video speed focusing (i.e., within $15 \mathrm{~ms}$ ) is achieved using a 2-D bandpass filter requiring only the summation and differentiation pixels without using multiplication operation, in the twodimensional convolution is necessary to estimate the power of the selected spectral bands.

Then, the image that fit the criterion of minimum focusing analysed to find the iris, with the precise localization of its boundaries with the use of strategies coarse-fine processing using a single-point assessment of the accuracy coordinates of the centre and radius as the iris and the pupil. Despite the fact that the iris search greatly limit the ability to search a pupil, the concentricity of these boundaries can not be accepted. Very often the centre of the pupil is in the nasal and lower parts of the iris. Its range may be from 0.1 to 0.8 the radius of the iris. Thus, all three parameters defining the pupillary circle must be assessed separately from all of the iris settings.

$300-\mathrm{MHz}$ computer processor with RISC reduced instruction set may be performed about 100,000 total comparisons between different irises per second such processor 1 or 1.7 million per second for the server at a frequency of $2 \mathrm{GHz}$. This is accomplished by comparing the effective implementation process of the elementary Boolean operators and $\otimes \cap$ with the terms acting in parallel on the calculated phase bit sequences. If the database contains many millions of registered persons, the inherent parallelism to speed the search process should be used, dividing the entire database into smaller parts, which must be sought in parallel. It must be ensured that the threshold of resolution adapted for each of the parallel search systems to ensure that no false matches will not be performed independently of each other. Mathematics iris recognition algorithms, in particular, the class of binomial distributions, which are generated when comparing different values make it clear that in the databases with the size of the total population, the search can be carried out in parallel to provide accurate and quick decisions.

To obtain high-quality images at the stage of registration is necessary to consider not only the conditions of natural light reception area, but the eye colour user. To solve this problem it is recommended to use biometric authentication system for iris with a functional visible and IR ranges of scanning, as shown in Fig. 4.

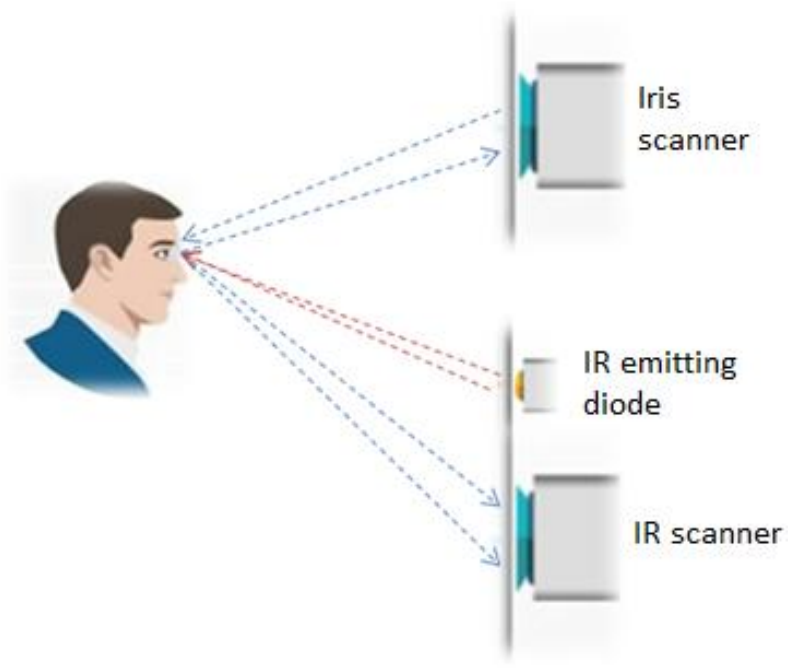

Fig. 4. Vector eye image registration using cameras with IR and visible lightning

Also, to improve the quality of the recorded image is necessary to prevent the dark noise of the sensor and the electronic camera apparatus originally, which we recommend:

1) Select high-quality sensors and electronic components for the camera;

2) Pay due attention to building a robust architecture of electronic components camera;

3) Prevent overheating of the sensor chamber and other analogue components; 
4) Take steps to prevent the effects of external noise, the background noise generated by a signal (e.g., via shielded cables).

After registering eye images, the system will evaluate the quality of the images obtained in the visible and IR ranges of scanning, and selects the image with the best quality for each band. Then, the image data is compared to the new third step of the recognition algorithm illustrated in Fig. 5, to select the most suitable image for subsequent isolation region thereon iris [9].

Thus, the system performs allocation of an iris in an image of the highest quality, which will satisfy the most not only the condition of lighting the reception area, but also the colour of the user's system eyes.

To assess changes in the quality of the image by using the biometric authentication system with iris functional visible and IR ranges scanning use Theorem multiplying the probabilities for independent events [6] according to formula (7):

$$
\mathrm{P}(\mathrm{AB})=\mathrm{P}(\mathrm{A}) \cdot \mathrm{P}(\mathrm{B}),
$$

where $\mathrm{P}(\mathrm{AB})$ - False Rejection Rate (FRR) probability of error for a biometric authentication system for iris with a functional visible and infrared scanning ranges; $\mathrm{P}(\mathrm{A})$ - the probability of errors in registration FRR Images in the visible range; $\mathrm{P}(\mathrm{B})$ - the probability of errors in the registration of FRR Images in the infrared range.

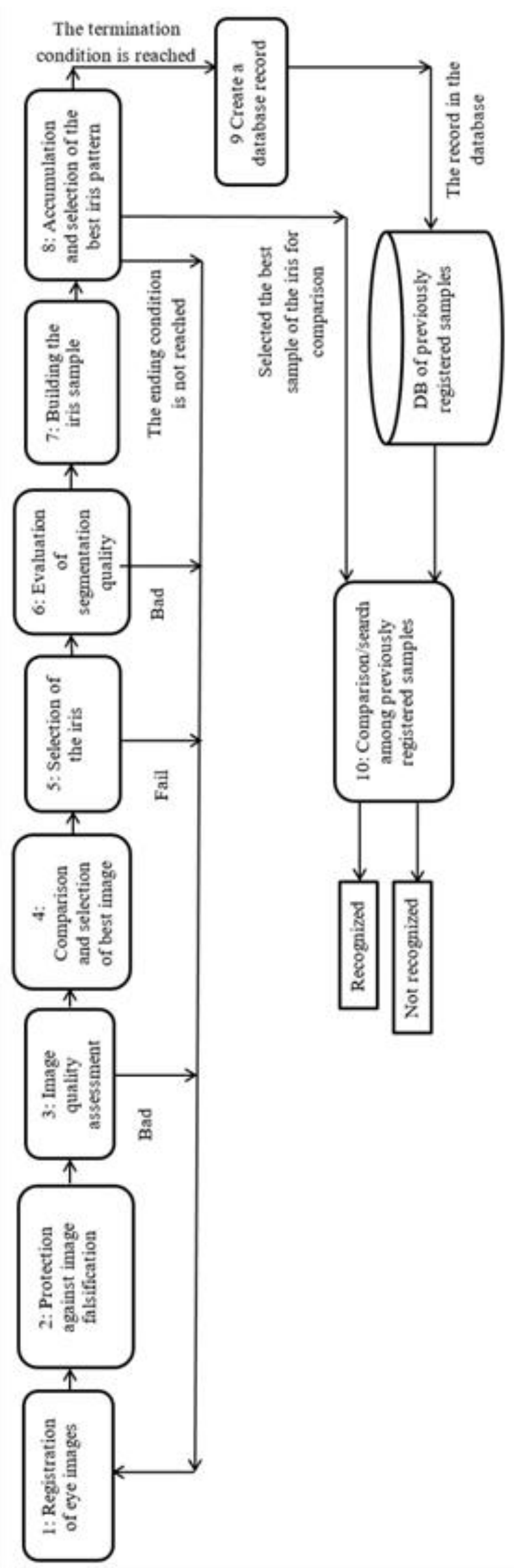

Fig. 5. Enhanced by iris recognition system 
Then FRR error probability for a biometric authentication system for iris with a functional visible and IR ranges of scanning by the formula (8) is:

$$
\mathrm{P}(\mathrm{AB})=0,771 \cdot 0,144=0,111,
$$

According to the obtained results, it can be concluded that by using the biometric authentication system for iris with a functional visible and IR ranges scan error of the first kind of FRR is now $11.1 \%$, which $66 \%$ less than when of registration images in the visible region and $3.1 \%$ in the IR. Reduced the likelihood occurrence of error FRR indicative of the image quality improvement, and thus to increase the accuracy of recognition for the entire system.

Since the correct operation of the system after each stage of the implementation of the fourth step in iris recognition algorithm using digital image treatment from the noise, it is possible to eliminate this deficiency by optimizing the use of digital filters [10] to increase the speed of the filters by using the optimal set of residual classes system (RCS) modules that will help compensate for the time required for improved image capture eyes [11].

\section{CONCLUSION}

To obtain high-quality images at the stage of registration is necessary to consider not only the conditions of natural light reception area, but the eye colour user. To solve this problem it is recommended to use a biometric authentication system for iris with a functional visible and infrared scanning range.

After registering eye images, the system will evaluate the quality of the images obtained in the visible and IR ranges of scanning, and selects the image with the best quality for each band. Then, the image data is compared to the new third step of the recognition algorithm to select the most suitable image for subsequent isolation region thereon iris.

Thus, the system performs allocation of an iris in an image of the highest quality, which will satisfy the most not only the condition of lighting the reception area, but also the colour of the user's system eyes.

It is estimated change image quality obtained when using a biometric authentication system for iris with a functional visible and IR scanning multiplication of probabilities theorem FRR error is now $11.1 \%$; $66 \%$ less than when of registration images in the visible region and $3.1 \%$ in the IR reduce the likelihood occurrence of error FRR indicative of the image quality improvement, and thus to increase the accuracy of recognition for the entire system.

However, the proposed recommendations lead to the addition of a new step in the recognition algorithm that increases the work of the entire algorithm as a whole.

To compensate for the time required for advanced imaging the eye, it is recommended to use the optimal set of RCS modules that enhance the performance and specifications digital filters in iris image processing systems.

\section{REFERENCES}

[1] Global Biometrics Market Revenue to Reach \$15.1 Billion by 2025 . (Source: https://www.tractica.com/newsroom/press-releases/globalbiometrics-market-revenue-to-reach-15-1-billion-by-2025/)

[2] Clyde W. Oyster. "The Human Eye: Structure and Function. Revised Edition", Sinauer Associates is an imprint of Oxford University Press, 1999. - $766 \mathrm{p}$

[3] Shlaev, D.V., Gaychuk, D.V., Rezenkov, D.N., Minkina, T.V., Durakova, A.S. "Development the module algorithm of information system to face detection for smart environments". Research Journal of Pharmaceutical, Biological and Chemical Sciences, 2016: Vol. 7, Issue 6, pp. 2299-2302 (in Russian).

[4] University of Tehran IRIS (UTIRIS). Iris image database. (Source: https://utiris.wordpress.com/).

[5] Daugman J. "How iris recognition works". J. Opt. Soc. Amer. A. 2004; Vol. 14, no. 1, pp. 21-30.

[6] Das, A., Pal, U., Ferrer, M.A., Blumenstein, M., Štepec, D., Rot, P., Emeršič, Ž., Peer, P., Štruc, V., Kumar, S.V.A., Harish, B.S. "SSERBC 2017: Sclera segmentation and eye recognition benchmarking competition". In: IEEE International Joint Conference on Biometrics, IJCB 2017, Vol. 2018-January, pp. 742-747.

[7] Rot, P., Emersic, Z., Struc, V., Peer, P. "Deep Multi-class Eye Segmentation for Ocular Biometrics". In: IEEE International Work Conference on Bioinspired Intelligence, IWOBI 2018. Article \# 8464133 .

[8] Lozej, J., Meden, B., Struc, V., Peer, P. "End-to-End Iris Segmentation Using U-Net". In: IEEE International Work Conference on Bioinspired Intelligence, IWOBI 2018. Article \# 8464213.

[9] Vrček, G., Peer, P. "Iris-based human verification system: A research prototype". In: 16th International Conference on Systems, Signals and Image Processing, IWSSIP. 2009. Article \# 5367801.

[10] Piestrak S. J. "A high-speed realization of a residue to binary number system converter". In: IEEE Trans. on Circuits and Systems-II: Analog and Digital Signal Processing, 1995, pp. 661-663.

[11] Younes D., Steffan P. "A comparative study on different modules sets in residue number system". In: International Conference on Computer Systems and Industrial Informatics (ICCSII). 2012, pp. 1-6. 